Draft Version September 22, 2021

Typeset using $\mathrm{LAT}_{\mathrm{E}} \mathrm{X}$ twocolumn style in AASTeX63

\title{
Correction of Radio Interferometric Imaging for Antenna Patterns
}

\author{
W. D. Cotton ${ }^{1}$ and T. Mauch ${ }^{2}$ \\ ${ }^{1}$ National Radio Astronomy Observatory \\ 520 Edgemont Road \\ Charlottesville, VA 22903, USA \\ ${ }^{2}$ South African Radio Astronomy Observatory, 2 Fir St., Observatory, South Africa
}

(Received ??; Revised ??; Accepted ?? draft September 22, 2021)

\section{Submitted to PASP}

\begin{abstract}
We describe and demonstrate a technique for correcting direction dependent artifacts due to asymmetries in antenna patterns and differences among antennas used in radio interoferometric imaging. The technique can correct images in all Stokes parameters I, Q, U and V and is shown with simulated data to reduce the level of artifacts to near the level of those from the basic imaging technique. The demonstrations use simulations of a mixed array of 13.5 and $15 \mathrm{~m}$ antennas with asymmetric patterns. The flux densities and spectral indices of the sources in a high dynamic range realistic simulated sky model are well recovered. Source polarization properties are also recovered in tests using unpolarized and partly polarized sources. The additional computational run time for Stokes I correction is about $50 \%$ in a realistic test described.
\end{abstract}

Keywords: Astronomical techniques, Interferometry, Polarimetry

\section{INTRODUCTION}

Radio interferometric arrays produce images of the sky as modified by the beam pattern of the individual antennas. Asymmetries in 2 axis alt-az mounted antennas introduce time dependent gain variations in the direction of sources off the axis as the antenna pattern rotates on the sky with parallactic angle ${ }^{1}$. This is the equivalent of observing a time varying source and will introduce non-convolutional artifacts in the derived image. The use of heterogeneous arrays, arrays of antennas with dissimilar patterns on the sky, are an even more extreme case as the different antennas see a different effective sky even at the same time. Artifacts from bright sources caused by beam asymmetries and differences among antennas can seriously corrupt the imaging of weaker sources. It is worth noting that accurate cor-

Corresponding author: William Cotton

bcotton@nrao.edu

${ }^{1}$ ASKAP has a 3 axis mount to eliminate this effect (Johnston et al. 2008). rection for beam models requires accurate pointing of the antennas.

Antenna design can optimize polarization performance over only a limited area of the antenna beam on the sky. Away from this limited area, the antennas introduce a spurious polarized response to total intensity. As source fractional polarization can be a few percent or less, this instrumental response can dwarf source polarization in regions of the antenna pattern with comparable, or larger, instrumental polarizations. Observations over a range of parallactic angle (observing geometry) tends to reduce, but not eliminate this effect. This effect can render polarization measurements of limited use over a significant portion of the, otherwise useful, antenna beam. An array with heterogeneous antenna designs further complicates this problem.

This paper describes a technique for correcting derived images for the effects of antenna patterns and presents tests using simulations of the heterogeneous array MeerKAT+ (13.5 m MeerKAT dishes plus $15 \mathrm{~m}$ SKA 
dishes) as implemented in the Obit package (Cotton $2008)^{2}$.

\section{DIRECTION DEPENDENT EFFECTS}

Direction dependent effects (DDEs) have long been recognized as a fundamental limitation to radio interferometric imaging. Direction dependent effects are always present in the form of the power gain pattern of the interferometer elements but in cases in which they are constant during the observations they can be dealt with using a "Primary beam correction" of the final image. This can be the case when the elements have an equatorial mount or a third axis "field rotator" as is used in ASKAP (Johnston et al. 2008).

Time variable direction dependent effects such as antenna pointing errors, circular asymmetries in altaz mounted antennas, variations in atmospheric phase across the field of view and the like will introduce image artifacts which will decrease the dynamic range and may obscure fainter objects in the presence of brighter ones. Mitigation of these are needed if they limit the science. A general discussion of DDEs with a formalism to describe them is given in Smirnov (2011a)

DDEs come in two basic flavors, those which must be inferred from the observations themselves and those which can be derived from models of the instrument or external measurements of the atmosphere. Techniques which derive the corrections from the data can, and have, been used to correct effects that could be modeled. Data sets contain a finite amount of information; calibration inferred from the data will use some of these "degrees of freedom" that could otherwise be used to improve the image quality. Correction based on external measurements is thus preferred to that derived from the data. In practice, both predictable and unpredictable effects will be present in most data.

\subsection{DDEs inferred from the observations}

Most attempts to date to correct DDEs have, of necessity, inferred the effects from the observations.

\subsubsection{Peeling}

The flux density distribution of radio sources is such that at lower frequencies, artifacts are frequently dominated by a few bright sources. These can be frequently greatly reduced using "Peeling" (Noordam 2004) in which the complex antenna gains in the direction of the bright sources are derived and the contribution of the peeled source subtracted from the data set. If multiple

2 http://www.cv.nrao.edu/ bcotton/Obit.html sources need peeling they are processed sequentially. An example of peeling is given in Cotton (2021).

\subsubsection{Differential gains}

A more sophisticated approach to peeling multiple sources is the "differential gain" method of Smirnov (2011a). Complex gains in the directions of multiple sources are solved for simultaneously. This reduces the buildup of numerical errors from sequentially peeling multiple sources.

\subsubsection{Clustered calibration}

Especially at low frequencies where the ionosphere is important, deriving the gains in the directions of a few bright sources may be inadequate. On the other hand, most sources are too weak to be individually detectable. The "Clustered calibration" described by Kazemi et al. (2013) divides the sky up into regions with sufficient number of sources that a set of collective gains can be derived. These gains are then used to calibrate and image the respective regions.

\subsubsection{Field based calibration}

A different approach to ionospheric calibration is "Field based calibration" of (Cotton et al. 2004; Cotton 2005) which is applicable in the regime (Lonsdale 2005) in which the geometry of the field of view is distorted but not on the scale of individual sources. Snapshot imaging of strong sources in the field allows time sampling of this distortion field which is fitted with a Zernike phase screen. This screen is used to dedistort/redistort the data during deconvolution. This technique has been applied to VLA data at 74 (Lane et al. 2014) and 327 (Uson \& Cotton 2012) MHz.

\subsection{5. $S P A M$}

A generalization of Field based calibration is the "SPAM" (Source Peeling And Modeling) of Intema et al. (2009) which fits a function to the phases derived from peeling the bright sources in the field. This should work even when the individual sources start becoming distorted.

\subsubsection{Facet Calibration}

A refinement of the Clustered calibration is the "Facet Calibration" of van Weeren et al. (2016). The initial direction independent calibration is used to subtract all sources in the initial CLEAN from the data and the field of view is divided into a compact set of facets. Sources from a given, brightest facet are added back and a single set of calibration parameters for that facet is derived. The facet's sources are then subtracted, correcting for these DDEs before moving to the next brightest facet. 


\subsection{Modeled DDEs}

At present, the DDEs that can be effectively modeled are the antenna gains which are modeled either by EM calculations or holography. This has been done for MeerKAT Asad et al. (2021) and the VLA Iheanetu et al. (2019) at L band.

\subsubsection{AW Projection}

Antenna gains are a multiplicative effect in the image domain hence are convolutional in the Fourier domain. The "AW projection" adaptation of "W projection" (Bhatnagar et al. 2008) by Bhatnagar et al. (2013) allows including corrections for time and direction dependent antenna gains in the convolution kernel used in gridding the data. These gains must then be unapplied in the degridding step when subtracting the sky model from the visibility data. An application to LOFAR is described in Tasse et al. (2013).

When ionospheric phase fluctuations are rapid, full AW projection gets very computationally expensive. van der Tol et al. (2018) describes a technique for reducing this cost.

\subsubsection{DDFacet}

An alternate approach to reducing the cost of AW projection is using image plane facets as described in Tasse et al. (2018) (DDFacet). DDFacet can apply full Jones externally defined models of antenna beam or other instrumental or atmospheric transmission effects. Corrections applied in imaging must be unapplied in the degridding step. An application of DDFacet to deep LOFAR imaging is given in Tasse et al. (2021).

\section{ANTENNA BEAM CORRECTIONS}

The technique developed in the following is to ignore the effects of antenna beam asymmetries and differences among antennas when making dirty/residual images in a CLEAN based deconvolution but then calculating and subtracting an accurate instrumental response to a partial sky model. This calculation uses the approximation that the sky model only includes the Stokes parameter being deconvolved.

In order to avoid numerical problems in regions of the image where the amplitude of the parallel hand antenna pattern is low, the ratio of the true antenna pattern to a "perfect" well behaved, symmetric, real beam is used to correct the instrumental response to the CLEAN sky model. After multiple major cycles, the residuals approach zero and the sky model approaches the true sky model modified by the "perfect" antenna pattern. Each major cycle needs to be fairly shallow to avoid incorporating artifacts into the CLEAN sky model.
This procedure can correct the spurious off-axis response to any I, Q, U or $\mathrm{V}$ but artifacts arising from Stokes I can seriously adversely affect the images of polarized emission so it should be done first. The final Stokes I sky model can then be used to subtract the array's response from the initial dataset which is then used to image $\mathrm{Q}, \mathrm{U}$ and $\mathrm{V}$. This procedure is then repeated for Stokes Q, U and V, subtracting the response to the final CLEAN model before moving on to the next Stokes parameter.

We adopt the formalism of Smirnov (2011b) to describe the direction dependent response of an interferometer. Assuming that the usual direction independent calibrations have been applied to the data and there are no direction dependent effects other than the beam shapes, the instrumental response for baseline $a-b$ in terms of $2 \times 2$ complex matrices becomes (Smirnov 2011b)

$$
V_{a b}(\nu, t)=\int_{l} \int_{m} E_{a, \nu, t, l, m}^{\prime} X_{\nu, t, l, m} E_{b, \nu, t, l, m}^{\dagger \dagger} d l d m / n,
$$

where $l$ and $m$ (with $n=\sqrt{1-l^{2}-m^{2}}$ ) are direction cosines; $E^{\prime}{ }_{a, \nu, t, l, m}$ and $E^{\prime}{ }_{b, \nu, t, l, m}$ are the ratios of the complex antenna beam responses in the direction of $(l, m)$ at time $t$ and frequency $\nu$ for the first and second antennas of the baseline to the "perfect" antenna beam pattern and ${ }^{\dagger}$ signifies the conjugate transpose. $X_{\nu, t, l, m}$ is given by

$$
X_{\nu, t, l, m}=B_{\nu, l, m} e^{-2 \pi\left(u_{\nu, t} l+v_{\nu, t} m+w_{\nu, t}(n-1)\right)} .
$$

$B_{\nu, l, m}$ is the "source coherence matrix" and $u_{\nu, t}, v_{\nu, t}, w_{\nu, t}$ are the spatial frequency coordinates of baseline $a-b$ at time $t$ and frequency $\nu$.

Replacing the continuous sky brightness with $N$ discrete sources Equation 1 becomes

$$
V_{a b}(\nu, t)=\sum_{j=1}^{N} E_{a, \nu, t, l_{j}, m_{j}}^{\prime} X_{\nu, t, l_{j}, m_{j}} E_{b, \nu, t, l_{j}, m_{j}}^{\prime \dagger} .
$$

The ratio of the true beam pattern for antenna $k$ (a or b) to the "perfect" beam corresponding to the position of component $\mathrm{j}$ at frequency $\nu$ and time $t$ can be expressed as

$$
E_{k, j, \nu, t}^{\prime} \quad=\quad\left[\begin{array}{cc}
p p_{k, j, \nu, t}^{\text {ratio }} & p q_{k, j, \nu, t}^{\text {ratio }} \\
q p_{k, j, \nu, t}^{\text {ratio }} & q q_{k, j, \nu, t, j}^{\text {ratio }}
\end{array}\right]
$$

where $p p^{\text {ratio }}, p q^{\text {ratio }}, q p^{\text {ratio }}$, and $q q^{\text {ratio }}$ are the ratios of the combinations of the two orthogonally polarized feeds. The variation in time is due to the parallactic angle change of alt-az mounted antennas tracking the source. 
The source coherency matrix for component $j, B^{j}$, for baseline $a-b$ at time $t$ and frequency $\nu$ for circular basis feeds is given by

$$
B_{a-b, \nu, t}^{j}=\frac{1}{2}\left[\begin{array}{cc}
I_{\nu}^{j}+V_{\nu}^{j} & Q_{\nu}^{j}+i U_{\nu}^{j} \\
Q_{\nu}^{j}+i U_{\nu}^{j} & I_{\nu}^{j}-V_{\nu}^{j}
\end{array}\right]
$$

where $I_{\nu}^{j}, Q_{\nu}^{j}, U_{\nu}^{j}$ and $V_{\nu}^{j}$ are the Stokes parameters of component $j$ and $i$ is $\sqrt{-1}$ and for linear feeds is given by

$$
B_{a-b, \nu, t}^{j}=\frac{1}{2}\left[\begin{array}{cc}
I_{\nu}^{j}+Q_{\nu}^{\prime j} & U_{\nu}^{\prime j}+i V_{\nu}^{j} \\
U_{\nu}^{\prime j}-i V_{\nu}^{j} & \left.I_{\nu}^{j}-Q_{\nu}^{\prime j}\right)
\end{array}\right]
$$

where

$$
\begin{aligned}
Q^{\prime j} & =Q_{\nu}^{j} \cos (2 \psi)+U_{\nu}^{j} \sin (2 \psi), \\
U^{\prime j} & =-Q_{\nu}^{j} \sin (2 \psi)+U_{\nu}^{j} \cos (2 \psi)
\end{aligned}
$$

and the parallactic angle $\psi$ is

$$
\psi=\tan ^{-1}\left(\frac{\cos \lambda \sin h}{\sin \lambda \cos \delta-\cos \lambda \sin \delta \cos h}\right) .
$$

Subtracting $V(\nu, t)$ (eq. 2) from the observed data will remove the response to the sky model including the spurious instrumental polarization terms. In a wide variety of cases, Stokes I dominates Q and U which in turn are much stronger than Stokes V. In this case Stokes I can be imaged and deconvolved with the approximation that $\mathrm{Q}, \mathrm{U}$ and $\mathrm{V}$ are zero. Once the response to Stokes $\mathrm{I}$ is removed, Q and $\mathrm{U}$ can be deconvolved with the approximation that $\mathrm{I}$ and $\mathrm{V}$ are zero.

\section{SIMULATIONS}

Simulations use the planned MeerKAT+ array which will include antennas located out to distances of nearly $10 \mathrm{~km}$ from its center at latitude -30:42:39.8, longitude $+21: 26: 38.0$. The array will be made up of a 'core' consisting of the original MeerKAT $13.5 \mathrm{~m}$ dishes whose locations are shown as plus signs in Figure 1. These will be supplemented by further dishes primarily at greater distance from the array center, these will have a $15.0 \mathrm{~m}$ diameter SKA design (circles in Figure 1). The different apertures of the MeerKAT and SKA dishes result in them having significantly different attenuation patterns. All antennas are equipped with feeds sensitive to linear polarization. Complex voltage patterns at $1510 \mathrm{MHz}$ derived from simulations of the MeerKAT antenna optics made by the EMSS (Asad et al. 2021) were provided by M. De Villiers and are shown in Figure 2.

The simulations described in this paper all use a pointing center at $\alpha=0^{h}, \delta=-80^{\circ}$, with a single 'track' of 120 time samples over a 12 hour observation period starting at 1970-01-01T15:50:15 UTC (when the array pointing has parallactic angle $\psi \sim 0^{\circ}$ ). The MeerKAT+

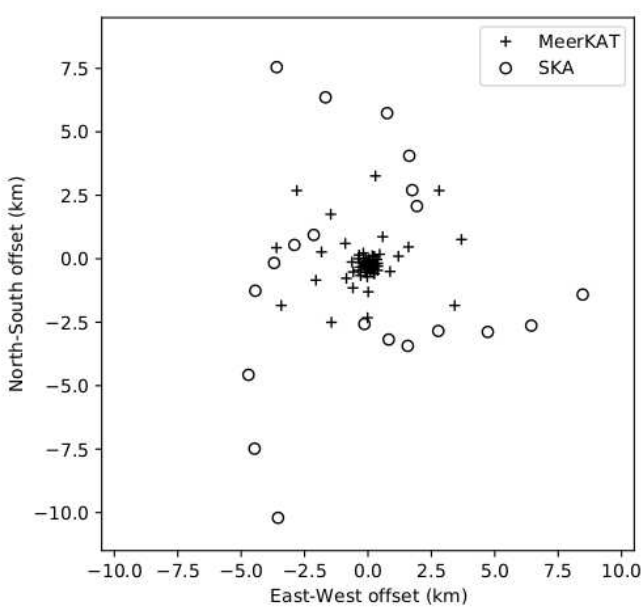

Figure 1. The MeerKAT+ array configuration used in the simulation. Positions of antennas are shown in $\mathrm{km}$ relative to the array center at latitude $-30: 42: 39.8$, longitude +21:26:38.0. The positions of the $6413.5 \mathrm{~m}$ diameter MeerKAT dishes are shown as plus signs and the $2015.0 \mathrm{~m}$ SKA dishes are shown as circles.

frequency range at L-band was sampled with 64 channels over the range $898.8-1669.2 \mathrm{MHz}$ and the 84 antennas with positions and types as shown in Figure 1.

\subsection{Simulated visibilities}

The simulation defines a function that computes complex visibilities given an observational setup and a list of point sources to be simulated. The visibilities are simulated using the Müller and Jones matrix formalism described by Smirnov (2011b) and references therein.

The flux density of any point source at a given right ascension $(\alpha)$ and declination $(\delta)$ is specified with a model describing the intrinsic Stokes $I(\nu), Q(\nu), U(\nu), V(\nu)$ flux densities of the source as a function of frequency $\nu$. These flux densities are then converted to linearly polarized coherencies at each channel frequency using Equation 5; this is also Equation 4.55 of Thompson et al. (2017). These intrinsic receptor coherencies are then formed into the $2 \times 2$ matrix

$$
B(\nu)=\left[\begin{array}{ll}
B_{\mathrm{XX}} & B_{\mathrm{XY}} \\
B_{\mathrm{YX}} & B_{\mathrm{YY}}
\end{array}\right]
$$

which is computed for each simulated channel with frequency $\nu$; the flux densities of simulated sources are assumed to be constant over time.

The antenna voltage responses of each linear polarization shown in Figure 2 will scale the intrinsic coherencies $B(\nu)$ of a point source differently for each timestamp and baseline. For an antenna $a$, the value of the voltage response at the position of the source at $(\alpha, \delta)$ with direction cosine coordinates $(l, m)$ relative to the phase 

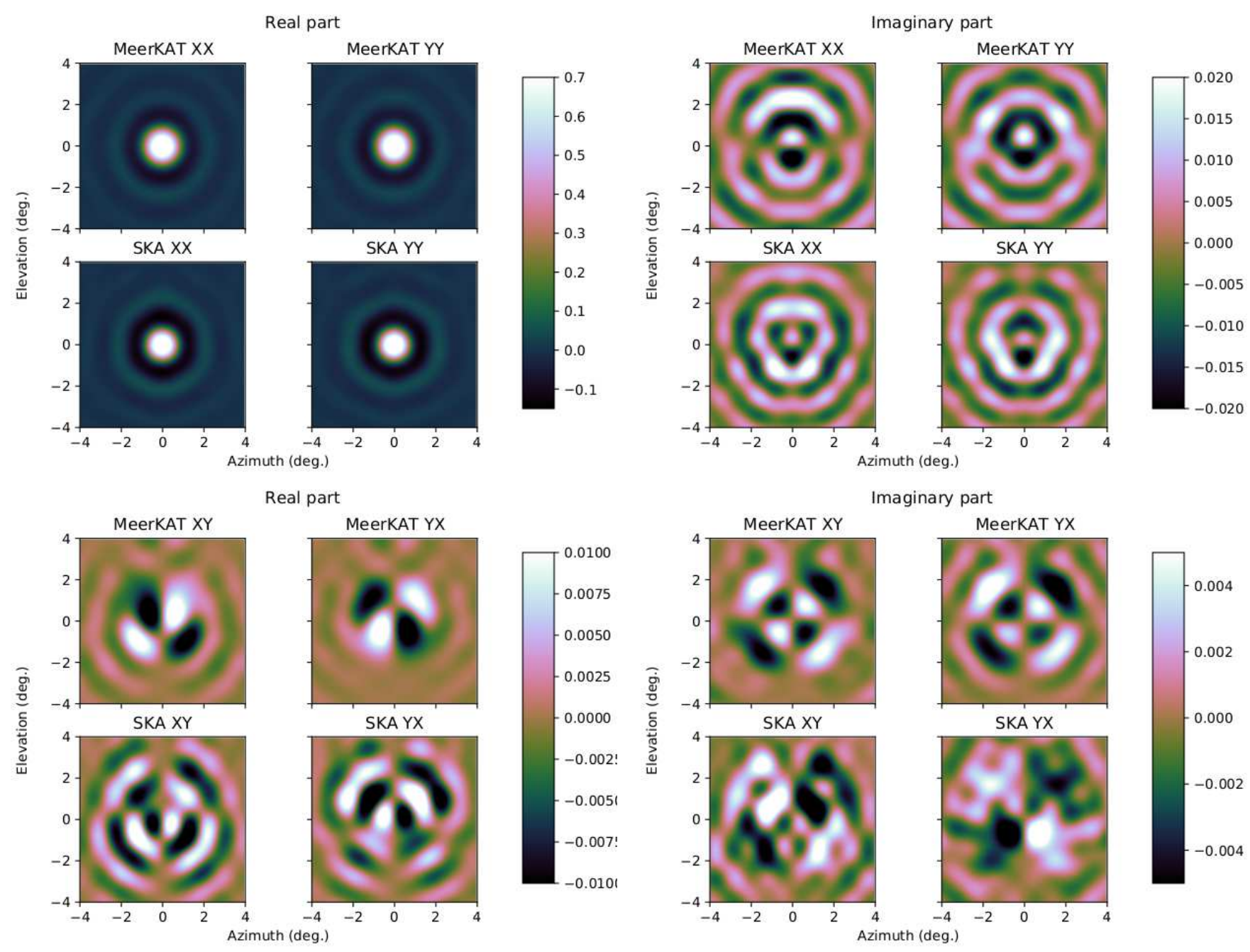

Figure 2. Real (left) and imaginary (right) parts of the complex voltage patterns at $1510 \mathrm{MHz}$ used in the simulation for $13.5 \mathrm{~m}$ MeerKAT dishes and $15.0 \mathrm{~m}$ SKA dishes (as labeled). The co-polarization response is shown in the top two rows and the cross-polarization in the bottom two.

center is scaled and rotated to coordinates $\left(l_{a}^{\prime}, m_{a}^{\prime}\right)$. The coordinates $\left(l_{a}^{\prime}, m_{a}^{\prime}\right)$ are derived from

$$
\left[\begin{array}{c}
l_{a}^{\prime}(t, \nu) \\
m_{a}^{\prime}(t, \nu)
\end{array}\right]=\left(\frac{\nu}{\nu_{\mathrm{ref}}}\right)\left[\begin{array}{cc}
\cos \psi_{a}(t) & \sin \psi_{a}(t) \\
-\sin \psi_{a}(t) & \cos \psi_{a}(t)
\end{array}\right]\left[\begin{array}{c}
l \\
m
\end{array}\right],
$$

where the fraction $\frac{\nu}{\nu_{\text {ref }}}$ radially scales the $(l, m)$ coordinates from the reference frequency $\left(\nu_{\text {ref }}\right)$ of the beam images in Figure $2(1510 \mathrm{MHz})$ to the frequency of interest and the source position is rotated (in an anti-clockwise direction) by the parallactic angle $\psi_{a}(t)$ of the antenna $a$ at time $t$.

The complex-valued beam voltages $E_{\mathrm{XX}}\left(l_{a}^{\prime}, m_{a}^{\prime}\right)$, $E_{\mathrm{XY}}\left(l_{a}^{\prime}, m_{a}^{\prime}\right), E_{\mathrm{YX}}\left(l_{a}^{\prime}, m_{a}^{\prime}\right)$ and $E_{\mathrm{YY}}\left(l_{a}^{\prime}, m_{a}^{\prime}\right)$ for an antenna are obtained from a bi-cubic interpolation of the beam voltage patterns in Figure 2. These are then formed into the $2 \times 2$ matrix

$$
E_{a}(t, \nu, l, m)=\left[\begin{array}{ll}
E_{\mathrm{XX}}\left(l_{a}^{\prime}, m_{a}^{\prime}\right) & E_{\mathrm{XY}}\left(l_{a}^{\prime}, m_{a}^{\prime}\right) \\
E_{\mathrm{YX}}\left(l_{a}^{\prime}, m_{a}^{\prime}\right) & E_{\mathrm{YY}}\left(l_{a}^{\prime}, m_{a}^{\prime}\right)
\end{array}\right],
$$

for each simulated timestamp $(t)$, frequency $(\nu)$ and antenna $(a)$. The coherency matrix describing the beamscaled flux density $F$ for a baseline between two antennas $a_{1}$ and $a_{2}$ and a single point source $i$ is then

$$
\begin{aligned}
& F\left(t, \nu, l, m, a_{1}, a_{2}\right)=E_{a_{1}}(t, \nu, l, m) \times B(\nu) \times \\
& E_{a_{2}}^{\dagger}(t, \nu, l, m) \times e^{-2 \pi j\left(u_{\nu} l_{i}+v_{\nu} m_{i}+w_{\nu}\left(n_{i}-1\right)\right)}
\end{aligned}
$$

where the term $E_{a_{2}}^{\dagger}$ represents the complex conjugate and transpose of the matrix $E_{a_{2}}$ and at a position given by direction cosines $\left(l_{i}, m_{i}, n_{i}=\sqrt{1-l_{i}^{2}-m_{i}^{2}}\right)$, on a baseline between antennas $a_{1}, a_{2}$ with UV-plane coordinates $\left(u_{\nu}, v_{\nu}, w_{\nu}\right)$ (measured in wavelengths at the given 
time and frequency). The flux density matrix $F$ describes the variation in the measured coherencies caused by differences in per-antenna voltage patterns in heterogeneous arrays (i.e. $F$ is a function of baseline, and if the antenna type of $a_{1}$ is MeerKAT and $a_{2}$ is SKA then $\left.E_{a_{1}} \neq E_{a_{2}}\right)$. It further includes the effect of variations due to beam asymmetries (i.e. $F$ is a function of time, and $E_{\mathrm{XX}}\left(l^{\prime}, m^{\prime}\right)$ etc. will vary as a source position rotates with parallactic angle in a non-symmetric voltage pattern).

When simulating $N$ point sources in the field the visibility for a given baseline, frequency and timestamp is

$$
V\left(t, \nu, a_{1}, a_{2}\right)=\sum_{i=1}^{N} \frac{F_{i}\left(t, \nu, l_{i}, m_{i}, a_{1}, a_{2}\right)}{n_{i}} .
$$

\section{SIMULATED EXAMPLES}

Simulated noiseless data sets were imaged in Obit Task MFBeam which was given the complex beam models described in Section 4 for the MeerKAT and SKA antennas in all four Stokes correlations (XX,YY,XY,YX) on a fine grid of frequencies. MFBeam uses faceting to correct for the "w" term and multiple frequency bins are imaged independently but CLEANed jointly to accommodate the spatially variant sky spectral distributions and antenna gain patterns. The interpolated locations in the beam patterns were updated whenever the parallactic angle changed by more than $0.25^{\circ}$. The "perfect" beam shape is a symmetrized version of that for MeerKAT (Mauch et al. 2020).

Since the positions of sources are generally in a random location in a facet and the dynamic range desired is high, the Obit autoCenter feature is used in which sources with peaks in excess of a given value, here 10 $\mathrm{mJy} /$ beam, are imaged centered in their own facets and "unboxes" are placed around the corresponding locations of other facets. The depth of any major cycle is restricted to no deeper than $75 \%$ of the initial peak residual flux density.

For comparison, the data were also imaged in the same way by MFImage in which beam corrections are not made; these are labeled "Uncorrected" in plots. A "Perfect" noiseless, dataset with no beam corruptions applied to the data was also imaged in MFImage to distinguish residual artifacts from the beam corrections from those due to the basic imaging. MFBeam and MFImage were run in an identical manner except for the calculation of the instrumental response to the CLEAN components.

Imaging was done on a Linux workstation running RHEL 7 with $16 \times \operatorname{Intel}(\mathrm{R}) \mathrm{Xeon}(\mathrm{R}) \mathrm{CPU}$ E5-2687W 0 @ $3.10 \mathrm{GHz}$ cores and 256 GByte of RAM, of which 100
GByte was configured into a RAM disk which was used for output and scratch files. This machine supports AVX but does not have a GPU.

\subsection{Realistic Sky}

The Square Kilometer Array Design Study (SKADS) developed a suite of simulations of the radio sky referred to collectively as the SKADS Simulated Skies $\left(\mathrm{S}^{3}\right)$. The $\mathrm{S}^{3}$ Semi-Empirical extra galactic $\left(\mathrm{S}^{3}\right.$-SEX; Wilman et al. (2008)) simulation was constructed by drawing radio sources at random from observed and extrapolated radio luminosity functions for various radio source populations and positioning them with regard to a realistic model of their spatial clustering. The $\mathrm{S}^{3}$ SEX galaxies simulation catalog contains positions in a sky area of $20^{\circ} \times 20^{\circ}$ and Stokes I flux densities derived from a model of the frequency dependence for each galaxy population at 5 frequencies between 0.151 and $18 \mathrm{GHz}$ to a limit of $10 \mathrm{nJy}$. A region containing 2401 galaxies within a $1^{\circ}$ radius to a $1400 \mathrm{MHz}$ flux density limit of $100 \mu \mathrm{Jy}$ was used to provide a realistic input sky model for generating noiseless visibilities with the heterogeneous MeerKAT+ configuration. Each of the simulated point sources was fitted with a spectrum including a spectral index and up to 2 curvature terms.

This realistic sky simulation was imaged with and without beam corrections. Imaging consisted of a CLEAN of 100,000 components reaching a depth of $\sim 10$ $\mu \mathrm{Jy} /$ beam using a loop gain of 0.1 . The beam corrections resulted in the effective beam being the MeerKAT, nominal, symmetric pattern (Mauch et al. 2020).

Without beam corrections, the off-source RMS was $2.58 \mu \mathrm{Jy} / \mathrm{bm}$ and with beam corrections $0.60 \mu \mathrm{Jy} / \mathrm{bm}$. The peak in the image is $0.55 \mathrm{Jy}$ so the nominal dynamic ranges over the whole field of view were $0.92 \times 10^{6}$ with beam correction and $0.21 \times 10^{6}$ without. Beam correction made further dynamic range improvements in the regions around bright sources. Cutouts around some of the stronger sources imaged by MFImage (no beam correction) are shown in Figure 3 Top Row and the same sources imaged by MFBeam (beam corrected) in Figure 3 Middle Row with the same stretch. The beam corrections greatly reduced the near in artifacts.

This simulation was also repeated except using the same, symmetric beam shape for all antennas which should not introduce artifacts. Imaging of this data with MFImage should show only the residual artifacts from imaging. Such results are shown in Figure 3 Bottom Row and should be compared with Figure 3 Middle Row. The bulk of the residual artifacts in Figure 3 Middle Row appear not to be the result of uncorrected beam 
effects. The RMS in the image from data without beam corruptions was $0.79 \mu \mathrm{Jy}$ beam $^{-1}$.

A comparison of the derived flux densities (Obit/FndSou) after a correction for the primary beam attenuation to the initial model values is shown in Figure 4. This figure shows that the flux densities in the input model are well recovered.

The input model consisted of point sources with a variety of spectra. The derived image had spectra fitted in each pixel with flux density at the reference frequency, spectral index and up to 2 curvature terms. The fitted spectral indices after correction for the primary beam gain are compared with the input model values in Figure 5 . The bulk of the fitted values are close to the input model values but with some scatter which increases for weaker sources and increasing distance from the pointing center.

Timing tests of imaging with corrections (MFBeam), without corrections (MFImage) and without beam corruptions (MFImageSym) were run as above but with a minimum flux density of $100 \mu \mathrm{Jy}$ beam $^{-1}$. These tests were identical except for the interferometer model calculation and the input data and are summarized in Table 1 .

CLEAN deconvolution is a nonlinear process and even slight differences can result in different paths to the solution. This is obvious from Table 1 in the varying amounts of work performed (columns "Comp.", "Major" and "Facet"). The difference in the run time between with and without correction is a function of 1) differences in the amount of work done, 2) differences in the cost of the interferometer model calculation and 3) the fraction of the total time used by the interferometer model calculation so the results in Table 1 are only suggestive. In this case the run time for applying the corrections is increased by $50 \%$ over not applying the corrections. The larger difference in the CPU times shows this to be quite sensitive to the number of $\mathrm{CPU}$ cores used, here 16 .

Further note that this test did not use GPU enhancement for the interferometer model calculation. Use of the GPU model calculation available for MFImage shows that it is so much faster than the CPU version that it frequently becomes a relatively insignificant fraction of the total run time (Cotton 2014). Similar gains are expected for a GPU version of the calculation used in MFBeam.

\subsection{Unpolarized Grid}

A second simulation used a sample of nine unpolarized sources on a grid covering the field of view to explore
Table 1. SKADS Timings

\begin{tabular}{lrrrrr}
\hline \hline Test & $\begin{array}{r}\text { clock } \\
\text { hr }\end{array}$ & $\begin{array}{r}\text { CPU } \\
\text { hr }\end{array}$ & Comp. & Major & Facet \\
& 2.96 & 23.19 & 11,347 & 88 & 11,955 \\
MFBeam & 2.00 & 9.97 & 13,562 & 77 & 10,630 \\
MFImage & 2.24 & 11.23 & 11,806 & 81 & 12,743 \\
\hline
\end{tabular}

Notes: "clock" = wall clock time, "CPU" = CPU time, "Comp." is the number of CLEAN components used, "Major" is the number of major CLEAN cycles and "Facet" is the number of facet imagings.

the spurious polarized response introduced by the beam shapes. This test consists of 2 parts:

\section{- Uncorrected}

These data used separate, asymmetric, MeerKAT and SKA beams and are corrupted by the beam effects. These corruptions were ignored by imaging in MFImage.

\section{- Corrected}

The same corrupted data were also imaged in MFBeam with corrections in the Stokes I. The resultant dataset with the corrections for the instrumental response were then imaged in Stokes Q, U and $\mathrm{V}$ without further correction.

The ratios of the maximum linear and circular polarization artifacts in the vicinity of each source to the total intensity flux density shown in Table 2 are plotted in Figure 6. As expected, the maximum fractional polarization artifacts are very low near the pointing center and increase with increasing distance. The corrections applied in this test reduced the maximum linearly polarized artifacts by approximately a factor of 30 and somewhat more for circular polarization. Our speculation is that since Stokes V is the difference in small values with linear feeds and $\mathrm{Q}$ and $\mathrm{U}$ are combinations of larger values, residual errors in the corrections will have a smaller effect on Stokes V.

\subsection{Polarized Grid}

A third simulation is similar to the second except that the grid of sources were each partially polarized. This test consists of 3 parts:

\section{- "Perfect" data}

The simulations used the same, symmetric beam patterns for all antennas. These should have no corruptions and were imaged by MFImage. Residual artifacts are the results of the imaging rather than limitations of the beam corrections. 

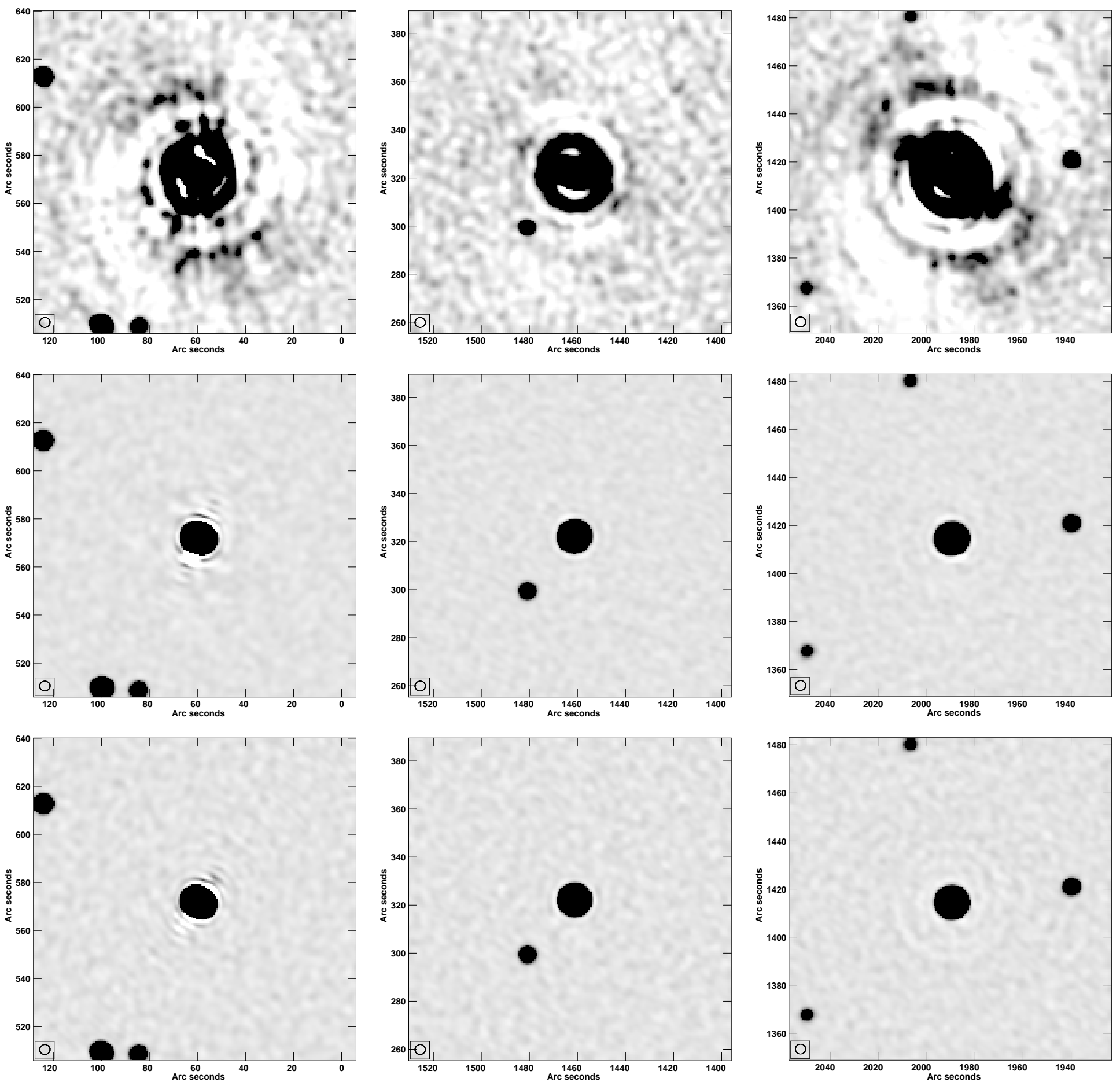

Figure 3. Reverse gray scale of the Stokes I CLEAN restored image in regions around 3 brighter sources in the SKADS simulation.

Top Row: Imaged without taking beam shapes into account. CLEANing was autoCentered with auto windowing. The pixel range displayed is $-5 \mu \mathrm{Jy}$ to $+15 \mu \mathrm{Jy}$. Off-source RMS noise is $2.58 \mu \mathrm{Jy} / \mathrm{bm}$. The resolution is shown in the box in the lower left.

Middle row: Like top row but taking beam shapes into account. Off-source RMS noise is $0.60 \mu \mathrm{Jy} / \mathrm{bm}$.

Bottom row: Like top row but with data simulated using a common, symmetric beam pattern, i.e. uncorrupted by beam shapes. Off-source RMS noise is $0.79 \mu \mathrm{Jy} / \mathrm{bm}$. 
Table 2. Unpolarized Grid Test Artifacts

\begin{tabular}{lrrrrrrrr}
\hline \hline Source & dist & $\begin{array}{r}\max \mathrm{I} \\
\mathrm{mJy} \mathrm{bm}^{-1}\end{array}$ & $\begin{array}{c}\mathrm{Q}_{\text {uncorr }} \mu \mathrm{Jy} \mathrm{bm}^{-1} \\
\mu \mathrm{Jy} \mathrm{bm}^{-1}\end{array}$ & $\begin{array}{c}\mathrm{Q}_{\text {corr }} \\
\mu \mathrm{Jy} \mathrm{bm}^{-1}\end{array}$ & $\begin{array}{c}\mathrm{U}_{\text {uncorr }} \\
\mu \mathrm{Jy} \mathrm{bm}^{-1}\end{array}$ & $\begin{array}{c}\mathrm{U}_{\text {corr }} \\
\mu \mathrm{Jy} \mathrm{bm}^{-1}\end{array}$ & $\begin{array}{c}\mathrm{V}_{\text {corr }} \\
\mu \mathrm{Jy} \mathrm{bm}^{-1}\end{array}$ \\
\hline $\mathrm{A}$ & 44 & 56.3 & $13.9-11.1$ & $2.2-0.4$ & $9.1-70.0$ & $0.4-0.3$ & $7.1-24.2$ & $0.9-0.1$ \\
$\mathrm{~B}$ & 30 & 70.9 & $9.0-42.1$ & $0.3-0.4$ & $21.1-14.6$ & $0.3-1.5$ & $6.8-24.0$ & $0.5-0.3$ \\
$\mathrm{C}$ & 44 & 56.2 & $29.3-21.3$ & $0.4-2.4$ & $84.7-15.6$ & $0.6-0.3$ & $16.8-30.7$ & $0.5-0.8$ \\
$\mathrm{D}$ & 31 & 70.9 & $39.4-5.1$ & $0.2-0.2$ & $14.1-10.1$ & $1.5-0.8$ & $7.0-10.6$ & $0.7-0.1$ \\
$\mathrm{E}$ & 0 & 94.3 & $1.0-1.2$ & $0.0-0.0$ & $1.7-1.3$ & $0.0-0.0$ & $0.5-1.3$ & $0.0-0.0$ \\
$\mathrm{~F}$ & 31 & 72.0 & $51.2-10.9$ & $0.3-0.2$ & $14.4-11.2$ & $1.4-0.3$ & $17.9-12.2$ & $0.4-0.6$ \\
$\mathrm{G}$ & 43 & 55.6 & $16.5-22.1$ & $0.3-2.1$ & $68.8-8.7$ & $0.4-0.6$ & $30.1-10.2$ & $0.7-0.2$ \\
$\mathrm{H}$ & 30 & 74.6 & $8.6-45.6$ & $0.4-0.2$ & $20.8-22.4$ & $0.3-1.4$ & $26.6-7.7$ & $0.5-0.3$ \\
$\mathrm{I}$ & 43 & 57.0 & $17.4-18.8$ & $2.2-0.4$ & $13.6-80.2$ & $0.5-0.4$ & $30.4-11.8$ & $0.7-0.8$ \\
\hline
\end{tabular}

Notes: "dist" is the distance from the pointing center, "Max I" is the peak Stokes I and the following columns are Stokes Q, $\mathrm{U}$ and $\mathrm{V}$ uncorrected and corrected for beam effects; each column contains the maximum and minimum within 1.1' in RA and dec. of each source. 

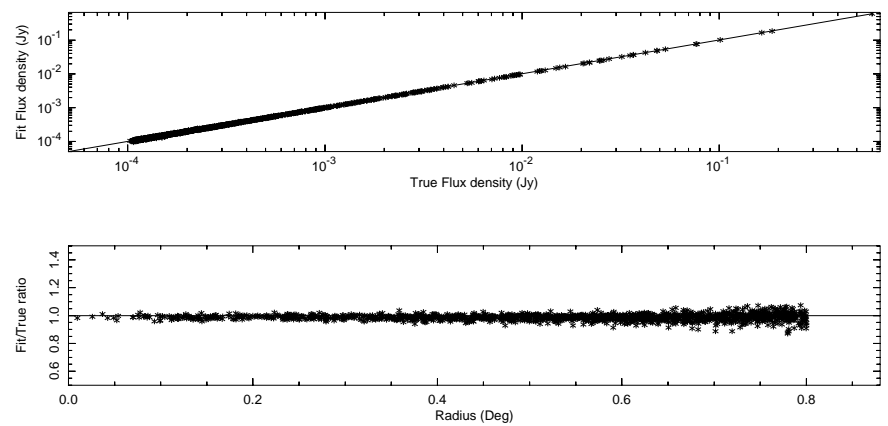

Figure 4. Comparison of recovered ('Fit') source flux densities to true values for the SKADS simulation. Top panel, the $\log$ "fitted" v. the log true values. The line shows the 1:1 values. The bottom panel gives the ratio of fit to true flux densities as a function of the distance from the pointing center.
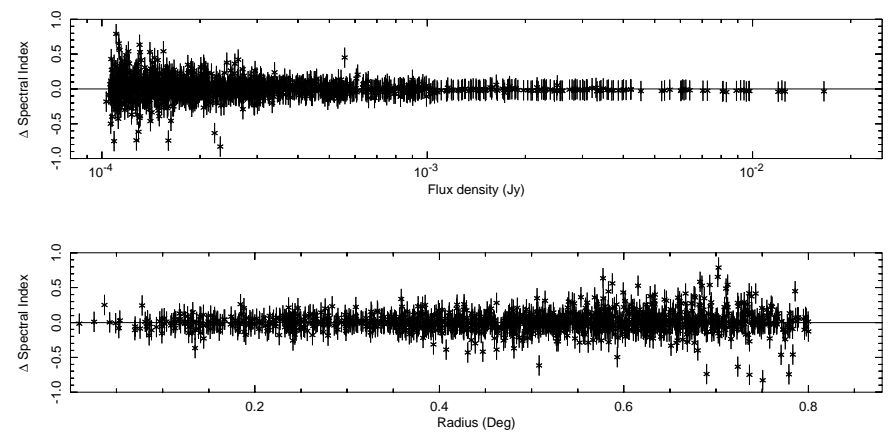

Figure 5. Difference between the fitted and true spectral index as a function of flux density and distance from the pointing center with fitting error bars for the SKADS simulation. Top panel, the fitted spectral index difference v. log flux density. The line shows the expected value (0.0). The bottom panel gives the spectral index difference as a function of the distance from the pointing center.

\section{- Uncorrected}

These data used separate, asymmetric, MeerKAT and SKA beams and are corrupted by the beam effects. These corruptions were ignored by imaging in MFImage.

\section{- Corrected}

The corrupted data were also imaged in MFBeam with corrections in the order, Stokes I, Q, U and V.

Residual images of these three processings are shown in Figures $7 \& 8$ for all four Stokes parameters for two of the sources. After MFBeam, the resultant images were primary beam corrected using the "perfect" beam shape and the fitted flux densities are compared with the model going into the simulation in Figure 9.

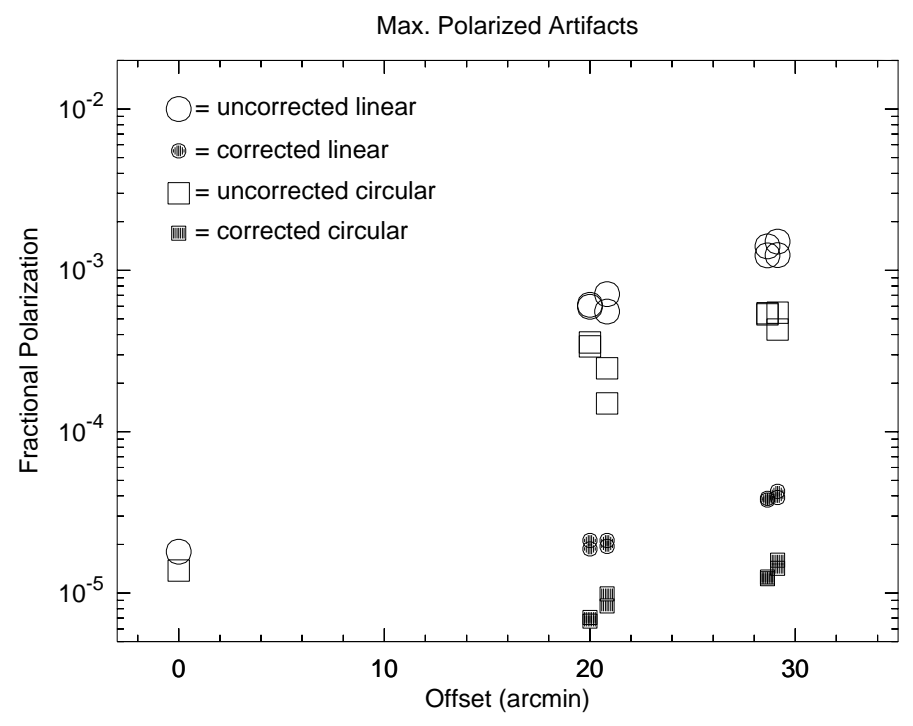

Figure 6. Maximum fractional polarization artifacts as a function of distance from the pointing center derived from Table 2. Open circles and squares are the uncorrected linear and circular polarizations and the filled symbols are those after beam corrections.

\section{DISCUSSION}

Asymmetries in, and differences among, antenna patterns in a radio interferometric array in an extended synthesis result in artifacts arising from brighter sources which are capable of obscuring fainter sources. These differences in antenna patterns will also result in spurious polarized responses to sources bright in total intensity (Stokes I). The preceding sections have explored examples of these and their corrections. The most important features of the process for correcting beam asymmetries and differences among antenna patterns are 1) how well the artifacts are reduced and 2) the relative expense of the computations.

\subsection{Accuracy}

The results of the realistic, SKADS, simulation shown in Figure 3 centered on several of the brightest sources which have very significant levels of artifacts when uncorrected. These artifacts are reduced to the level of those in images derived from data without the beam shape corruptions added. The overall "noise" level in the image was reduced by over a factor of four in this test. Figures $4 \& 5$ show that the flux densities of sources at all flux density levels were well recovered and the spectral indices of the sources recovered do not show a significant bias.

Spurious polarization responses to Stokes I generally are very small at the pointing center and increase away from it. The test using the grid of unpolarized sources 

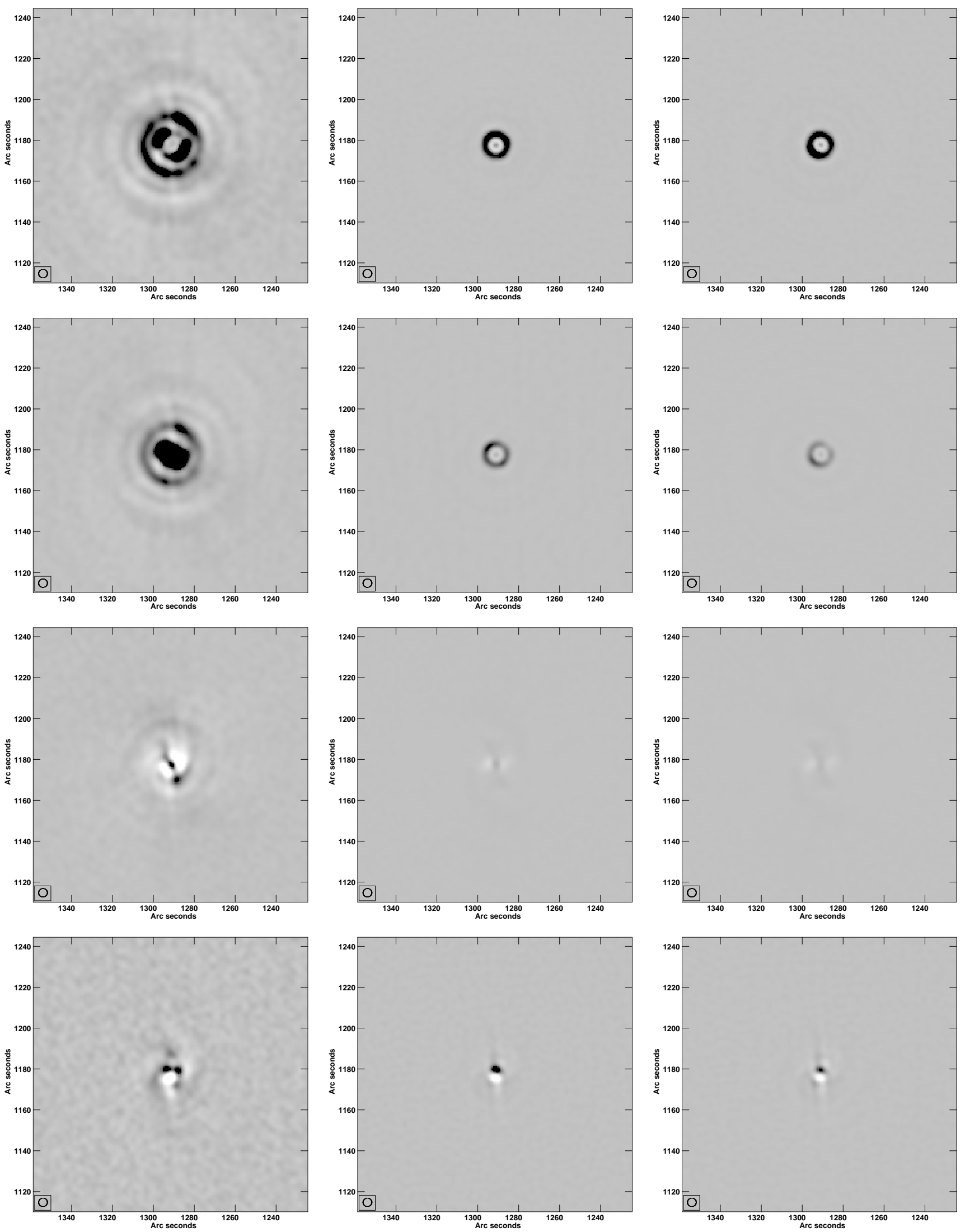

Figure 7. Reverse gray scale of the residuals around source A. Left column is the uncorrected image, center column is with corrections and the right column is the "Perfect" image. Top row is Stokes I, second row is Stokes Q, third row Stokes U and bottom row is Stokes V. The Stokes I pixel range displayed is $\pm 50 \mu \mathrm{Jy}$,Stokes Q \& U, $\pm 25 \mu \mathrm{Jy}$, and for Stokes V $\pm 10 \mu \mathrm{Jy}$. The resolution is shown in the box in the lower left. 

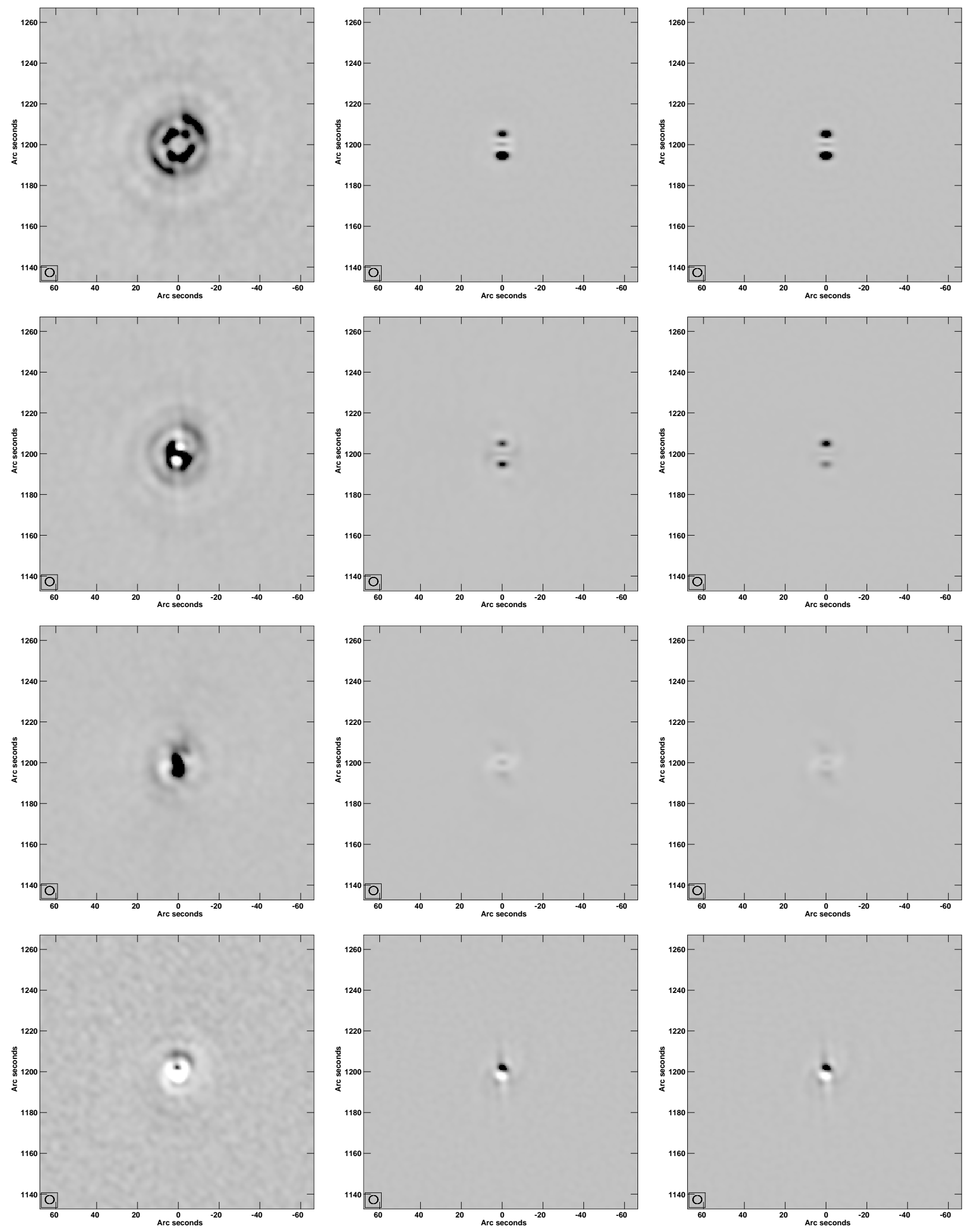

Figure 8. Like Figure 7 but source B. 


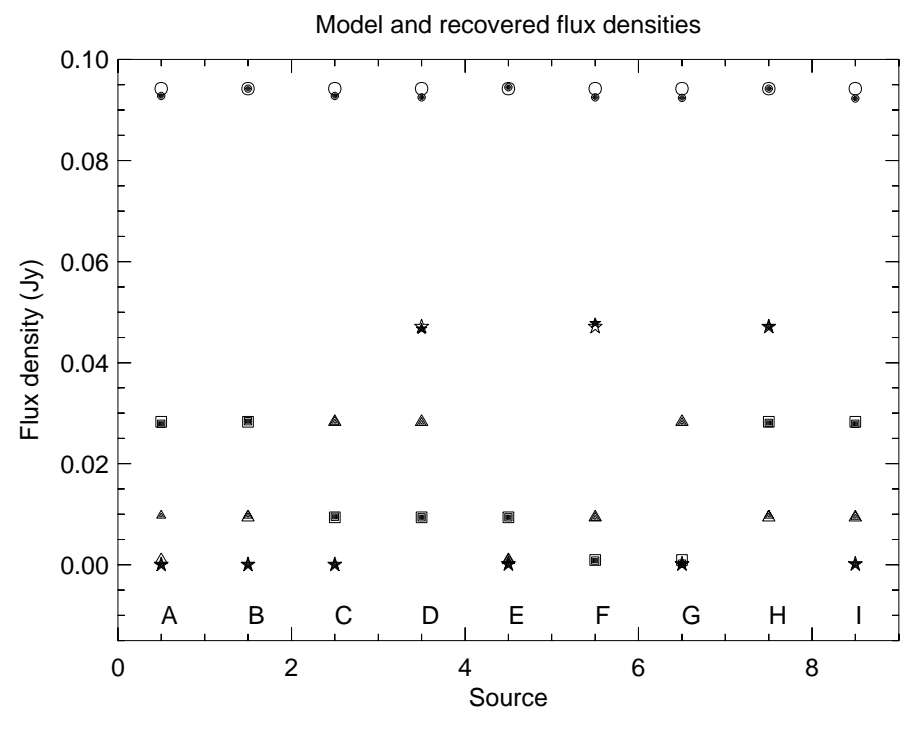

Figure 9. Comparison of simulation model flux densities and those recovered from imaging. Sources are numbered A-I, open symbols are the input model and filled symbols are the values recovered from imaging. Stokes I is a circle, Stokes Q a square, Stokes U a triangle and Stokes V a star.

in Table 2 and Figure 6 shows that the corrections reduce spurious linearly polarized artifacts at the edge of the field of imaged from $\sim 1.0 \times 10^{-3}$ to $\sim 4.0 \times 10^{-5}$ and spurious circular polarization drops from $\sim 6.0 \times 10^{-4}$ to $\sim 1.0 \times 10^{-5}$.

The test using the grid of partially polarized sources, Figures 7 - 9, shows that polarized artifacts were reduced to the level of those in the uncorrupted data and that the polarized flux densities of the input models are well recovered. This is true for sources with a significant range of fractional polarizations.

\subsection{Performance}

The CLEAN deconvolution is a very nonlinear process and slight differences can cause it to take rather different paths; this makes comparing the run times of tests with different algorithms tricky. The realistic sky simulation described in section 5.1 gives the best estimate of the increased cost of the beam correction described here. Imaging the corrupted data with beam corrections took $50 \%$ longer ( $2.96 \mathrm{v} 2.00$ hours) than without.

This test was performed using a CPU implementation of the model calculations as a GPU implementation is not yet available for the model with beam corrections and the machine used for the test did not have a GPU. GPU implementation of the sky model calculation dramatically changes the economics of imaging such that it usually becomes a minor component of the overall imaging computation.
The interferometer response to a given sky model does need to be accurately calculated, perhaps more so when the corrupting effects are not corrected in the imaging. In the technique described here, this is done via a "DFT" model calculation requiring large numbers of sine/cosine calculations. While these computations dominate the floating point operations of the imaging process, they are highly parallelizable; there are no dependencies which allows very efficient use of vector (SSE/AVX), multithreading and especially GPU implementations. GPU implementations of the DFT model calculation can reduce their cost to a small fraction of the total run time.

\subsection{Comparison with other Techniques}

The principle difference between the technique described here and previous methods of correction of beam effects described in Section 2.2 is that no attempt is made to correct the effects in the image formation. Instead, the calculation of the response to the sky model subtracted from the visibility data in each major CLEAN cycle includes these beam effects. As the process proceeds, the visibility/image residuals approach zero and the accumulated sky model approaches the desired one.

In the regime that the image corruptions are relatively minor, amplitude only ones with only small distortions, and no shift of source positions, the correction of dirty/residual images formed during deconvolution appears unnecessary. We note that effects such as those from the ionosphere which are largely in phase can, and do, introduce time dependent shifts in apparent position. These effects may seriously distort sources and thus must be corrected in the image formation. Techniques for correcting dirty/residual images such as "A Projection" can become quite expensive and yet still require corrections to be made in calculating the interferometer response to the sky model. The expense appears unwarranted in at least an interesting range of use cases.

"Degridding" of Fourier transforms of sky models is frequently done by interpolation of $2 \mathrm{D}$ complex grids at the spatial frequencies of the visibility measurements. The "DFT" method described here allows a much more accurate calculation of the instrumental response for each visibility measurement than interpolation from a grid. A relatively arbitrary model of the instrumental response can be calculated. While the DFT modeling requires a substantially larger number of floating point operations, the lack of dependencies allows efficient usage of modern computing hardware (SIMD instructions, threading, GPU) largely eliminates the runtime differences. 
Faceted imaging has a computational advantage over full field techniques such as $\mathrm{W}$ stacking or $\mathrm{W}$ projection in that many facets can be gridded and imaged in parallel using the same input data. Furthermore, once the full field has been imaged, only facets with emission likely to be CLEANed need be imaged in a given major cycle.

\section{SUMMARY}

At low frequencies and with small antennas, the field of view of radio interferometers is quite large and with the sensitivity of modern arrays, sources can be detected out into the side-lobes of the antenna pattern where there are usually significant asymmetries in the antenna gain. For 2 axis antennas with alt-az mounts this pattern rotates on the sky causing apparent brightness variations in the sources. This causes artifacts in the images which are non convolutional and, if uncorrected, can limit the dynamic range of the image. Heterogeneous arrays are an even more extreme case.

Furthermore, the spurious polarized instrumental response of the antennas increases with radius from the pointing direction and is also asymmetric. The spurious polarized response to Stokes I may corrupt, or even swamp, true polarized emission. Rotation of the pattern with parallactic angle will tend to reduce the instrumental polarization but generally not eliminate it.

A technique for making wide area beam full polarization corrections is described and shown to be effective for full polarization imaging when used in a CLEAN-like deconvolution. The general technique is to ignore beam effects when making dirty/residual images but then using an accurate beam model in computing the instrumental response to a partial sky model to be subtracted from the residual data before computing the next residual image. After multiple major cycles, the residual data converges towards zero and the accumulated sky model converges towards the correct one.

For wide area imaging a critical part of the technique is to correct the instrumental response from the actual, poorly behaved one(s), to a single well behaved, "perfect", i.e. real, symmetric, one. This avoids amplifying the noise in parts of the image in which the primary beam gain is low but which contains strong sources needing to be included in the deconvolution. For an array using multiple antenna designs with multiple diameter antennas, using the "perfect" beam of the smaller antenna seems to give better results.

A number of tests using simulated data show that the technique is effective and relatively efficient; with GPU implementation the additional cost of the sky model calculation can be reduced to a minor component of the imaging cost. A test of a realistic sky shows that high dynamic range, full field imaging is possible even with the heterogeneous array used for the imaging. The source flux densities and spectral indices of the input model are well recovered (Figures 4 \& 5).

Spurious polarized responses due to beam effects were greatly reduced as is shown in Table 2 and Figure 6 . Beam artifacts around polarized sources were reduced to the level of the basic imaging, see Figures $7 \& 8$ and model polarized flux densities are well recovered, see Figure 9.

The tests described here are for the MeerKAT+ array under development which includes two antenna designs, 1) MeerKAT $13.5 \mathrm{~m}$ dishes and 2) SKA design $15 \mathrm{~m}$ antennas. With the field of view and dynamic range requirements of this array and the very different antenna patterns, beam corrections will be needed all, or nearly all, of the time. This is an extreme case but a solution that works for it should also work for a homogeneous array with asymmetric antennas.

\section{ACKNOWLEDGMENTS}

The MeerKAT telescope is operated by the South African Radio Astronomy Observatory, which is a facility of the National Research Foundation, an agency of the Department of Science and Innovation. The National Radio Astronomy Observatory is a facility of the National Science Foundation, operated under a cooperative agreement by Associated Universities, Inc. We would like to thank the anonymous referee for encouraging a discussion in a larger context, leading to an improvement in the document.

Facilities: MeerKAT
Software: Obit Cotton (2008)

\section{REFERENCES}

Asad, K. M. B., Girard, J. N., de Villiers, M., et al. 2021, MNRAS, 502, 2970, doi: 10.1093/mnras/stab104

Bhatnagar, S., Cornwell, T. J., Golap, K., \& Uson, J. M. 2008, A\&A, 487, 419, doi: 10.1051/0004-6361:20079284
Bhatnagar, S., Rau, U., \& Golap, K. 2013, ApJ, 770, 91, doi: 10.1088/0004-637X/770/2/91 
Cotton, W. D. 2005, in Astronomical Society of the Pacific Conference Series, Vol. 345, From Clark Lake to the Long Wavelength Array: Bill Erickson's Radio Science, ed. N. Kassim, M. Perez, W. Junor, \& P. Henning, 337

Cotton, W. D. 2008, PASP, 120, 439, doi: 10.1086/586754

Cotton, W. D. 2014, Obit Development Memo Series, 35, 1. ftp://ftp.cv.nrao.edu/NRAO-staff/bcotton/Obit/GPUDFTv2.pdf

—. 2021, Obit Development Memo Series, 72, 1. ftp://ftp.cv.nrao.edu/NRAO-staff/bcotton/Obit/ManualPeel2.pdf

Cotton, W. D., Condon, J. J., Perley, R. A., et al. 2004, in Society of Photo-Optical Instrumentation Engineers (SPIE) Conference Series, Vol. 5489, Ground-based Telescopes, ed. J. Oschmann, Jacobus M., 180-189, doi: $10.1117 / 12.551298$

Iheanetu, K., Girard, J. N., Smirnov, O., et al. 2019, MNRAS, 485, 4107, doi: 10.1093/mnras/stz702

Intema, H. T., van der Tol, S., Cotton, W. D., et al. 2009, A\&A, 501, 1185, doi: 10.1051/0004-6361/200811094

Johnston, S., Taylor, R., Bailes, M., et al. 2008, Experimental Astronomy, 22, 151, doi: 10.1007/s10686-008-9124-7

Kazemi, S., Yatawatta, S., \& Zaroubi, S. 2013, MNRAS, 430, 1457, doi: 10.1093/mnras/stt018

Lane, W. M., Cotton, W. D., van Velzen, S., et al. 2014, MNRAS, 440, 327, doi: 10.1093/mnras/stu256

Lonsdale, C. J. 2005, in Astronomical Society of the Pacific Conference Series, Vol. 345, From Clark Lake to the Long Wavelength Array: Bill Erickson's Radio Science, ed. N. Kassim, M. Perez, W. Junor, \& P. Henning, 399

Mauch, T., Cotton, W. D., Condon, J. J., \& et al. 2020, ApJ, 888, 61, doi: 10.3847/1538-4357/ab5d2
Noordam, J. E. 2004, in Society of Photo-Optical Instrumentation Engineers (SPIE) Conference Series, Vol. 5489, Ground-based Telescopes, ed. J. Oschmann, Jacobus M., 817-825, doi: 10.1117/12.544262

Smirnov, O. M. 2011a, A\&A, 527, A107, doi: 10.1051/0004-6361/201116434

-. 2011b, A\&A, 527, A106,

10.1051/0004-6361/201016082

Tasse, C., van der Tol, S., van Zwieten, J., van Diepen, G., \& Bhatnagar, S. 2013, A\&A, 553, A105, doi: 10.1051/0004-6361/201220882

Tasse, C., Hugo, B., Mirmont, M., et al. 2018, A\&A, 611, A87, doi: 10.1051/0004-6361/201731474

Tasse, C., Shimwell, T., Hardcastle, M. J., et al. 2021, A\&A, 648, A1, doi: 10.1051/0004-6361/202038804

Thompson, A. R., Moran, J. M., \& Swenson, George W., J. 2017, Interferometry and Synthesis in Radio Astronomy, 3rd Edition (Springer), doi: 10.1007/978-3-319-44431-4

Uson, J. M., \& Cotton, W. D. 2012, Comptes Rendus Physique, 13, 14, doi: 10.1016/j.crhy.2011.10.016 van der Tol, S., Veenboer, B., \& Offringa, A. R. 2018, A\&A, 616, A27, doi: 10.1051/0004-6361/201832858 van Weeren, R. J., Williams, W. L., Hardcastle, M. J., et al. 2016, ApJS, 223, 2, doi: 10.3847/0067-0049/223/1/2

Wilman, R. J., Miller, L., Jarvis, M. J., et al. 2008, MNRAS, 388, 1335, doi: 10.1111/j.1365-2966.2008.13486.x 